Casanueva $V$, y cols.

Rev. Chil. Pediatr. 66 (4); 200-203, 1995

\title{
Concentración sérica de apolipoproteínas A-I y B en niños y adolescentes
}

\author{
Víctor Casanueva E. ${ }^{\dagger}$; Ximena Cid C. ${ }^{2}$; Cristián Milos G. ${ }^{3}$; M. Teresa Chiang S. ${ }^{4}$; \\ Celia Ruiz M. ' ; Pilar Marivil F. ${ }^{5}$; Paulina Casanueva C. ${ }^{6}$
}

\section{Serum concentrations of apolipoprotein A-I and B in children and adolescents}

Serum concentrotion of apolpoproteits A.l tapo A.tl and B lapo B) were cssoyed by an inmunenephelometric method 17 ;OO schoo, ci-ildren aged 5 lo 18 years $\$ 52$ boys and 48 gilsi randomly chosen from a previous sludy of $220 \hat{\mathrm{C}}$ chilidren. Mear serum apo $\mathrm{A} \cdot \mathrm{l}$ and apo $\mathrm{B}$ ccncentrations were $130 \pm 20 \mathrm{mg} / \mathrm{dl} \mathrm{cnd} 70 \pm 21 \mathrm{mg} / \mathrm{dl}$ respectively. Corresponding ralios were apo B/apo A-i =0,70; apo Avl/apo B = 1,90; LDL/apo B = 1.56. Fifth und 95 th percentile values for opo A.l and $000 \mathrm{~B}$ were 90 anc $170 \mathrm{mg} / \mathrm{dl}$ and 42 and $105 \mathrm{mg} / \mathrm{dl}$ respectively. Reference velues of serum apolipcproteins $A$-i and $B$ in chilean children moy be important as an inital slep to followup of coronary hearr disease precursors

[Key words: apolipoprotein A.l, apolipoprotein B.]

La hipercolesterolemia puede, por si sola, asociarse a lesiones vasculares ateroescleróticas'. Las alteraciones de las concentraciones séricas de colesterol, así como las del colesterol ligado a lipoproteina de alta (C-HDL) y de baja densidad (C-LDL), como factores de riesgo de enfermedades cardiovasculares (ECV), podrían ser detectadas a edad temprana. En escolares de Concepción, de 6 a 15 años de edad, $10 \%$ tienen concentraciones de colesterol total en el suero sobre $190 \mathrm{mg} / \mathrm{dl}$ y el C-LDL sobre $130 \mathrm{mg} / \mathrm{dl}$, ambas cifras consideradas de riesgo ${ }^{2}$. La sola determinación de colesterol y sus fracciones no es suficiente para predecir cl riesgo de enfeme-

1. Departamento de Pediatría, Facultad de Medicina, Universidad de Concepción.

2. Bioquímico. Laboratorio Central. Hospital Guillermo Grant Benavente, de Concepción.

3. Químico Farmacéntico. Departamento de Química Clínica e Inmunología, Facultad de Farmacia, Universidad de Concepción.

4. Bioguímico. Departamento de Fisiopatología, Facultad de Ciencias Biológicas, Universidad de Concepción

5. Licenciada en Bioquímica, Facultad de Farmacia, Universidad de Concepción.

6. Alumna de Carrera de Medicina. Universidad de Concepción.

Proyecto financiado por la Dirección de Investigación. Universidad de Concepción, $N^{\circ} 928812-1$. dad coronaria. pues ellos no son selectivos o suficientemente específicos. Las apolipoproteínas, ubicadas en la superficie de las lipoproteinas, tienen mayor precisión para estos efectos en adultos y niños 3.4 .

De las apolipoproteínas, las más importantes son la B (apo B) y la A-I (apo A-I). La primera es constituyente exclusiva de las lipoproteínas de muy baja y baja densidad (VLDL y LDL respectivamente) y su función es iniciar el transporte de los lípidos hacia los tejidos periféricos. La apo A-I está asociada a las lipoproteínas HDL y una de sus funciones es activar la lecitina colesterol aciltransferasa (LCAT), enzima clave en el transporte en reversa del colestetol desde los tejidos hacia el hígado ${ }^{5}$. A continuación se describen los perfiles de apo A-I y apo $B$ en niños y adolescentes de la ciudad de Concepción, octava región de Chile, con el proposito de expresar valores de referencia como complemento al estudio de su perfil lipídico.

\section{Material y Método}

Se estudiaron 100 niños y adolescentes -de los cuales 52 eran varones- entre 5 y 18 antos de edad, seleccionados aleatoriamente de un universo de 2200 escolares evalua- 
dos previamente, dentro de un programa de estudio de factores de riesgo cardiovascular en la población pediátrica. En cada uno se hicieron mediciones séricas de lípidos, tipoproleinas y apolipoproteínas A-l y B. Los lípidos y las lipoproteínas se determinaron con métodos ya descritos? Las apolipoprotefnas se midieron con un juego de análisis de Orion Diagnostica Turbox TM Espoo. Finlandia, mediante inmunoprecipitación en fase líquida y detección nefelométrica del punto final. El antisuero para apolipoproteína A-J 0 B se diluyó en tampón fosfato y se le agregó a una alicuota de suero a analizar. Previa incubación, se midió la dispersión de la luz producida por el complejo antígeno-anticuerpo. Esla última fue directamente proporcional a la concentración de apolipoproteína A-I o B de la inuestra ${ }^{6}$. El andilisis estadistico y de correlación se realizo con el frograma estadistico Systal.

\section{Resultados}

Se obtuvieron las siguientes concentraciones (expresadas como promedios y desviaciones estándar): colesterol total: $165 \pm 24,5 \mathrm{mg} / \mathrm{dl}$; CHDL: $49 \pm 10,6 \mathrm{mg} / \mathrm{dl}$; C-LDL: $99 \pm 23,5 \mathrm{mg} /$ dl; triglicéridos: $84 \pm 41,2 \mathrm{mg} / \mathrm{dl}$; apo A-I: 130 $\pm 26 \mathrm{mg} / \mathrm{dl}$ y apo B: $70 \pm 21 \mathrm{mg} / \mathrm{d}$. Los cuocientes resultantes fueron: apo B/apo $\mathrm{A}-\mathrm{I}=$ 0,70 ; apo $\mathrm{A}-\mathrm{I} / \mathrm{apo} \mathrm{B}=1,95$ y LDL/apo $\mathrm{B}=$ 1,56 .

Los coeficientes de correlación de Pearson entre las apolipoproteínas A-I y B con los lípidos y colesterol de lipoproteinas séricas mostraron una correlación positiva entre apo A-I y CHDL (r: 0,49) y débil entre apo A-I y colesterol total ( $r: 0,36$ ), no encontrándose correlación entre la apo A-I y C-LDL (r: 0,18 ) ni triglicéridos ( $r$ : -0.06). En el caso de la apo B existe correlación positiva mayor con C-LDL (r: 0,56$)$ y con el colesterol total (r: 0.55 ), no existiendo correlación con C-HDL (r: $-0,13)$ ni con triglicéridos (г: 0,21$)$.

En la tabla se expresan los percentiles de las concentraciones séricas de apo A-I y $\mathrm{B}$, siendo e] percentil 5 para apo A-I igual a $90 \mathrm{mg} / \mathrm{dl}$ y el percentil 95 para la apo $B$ de $105 \mathrm{mg} / \mathrm{dl}$, ambos establecidos como guías para identificar indjviduos que en riesgo potencial de desarrollar cardiopatía coronaria en su vida adulta.

\section{Comentario}

Las concentraciones séricas de las apolipoproteínas A-I y B de esta muestra son similares a las descritas por otros autores, aunque un poco menores. En el estudio de Bogalusa, con la técnica de electroinmunoensayo las concentraciones de apo A-I eran $139 \pm 21 \mathrm{mg} / \mathrm{dl}$ y de apo B $86,4 \pm 4,6 \mathrm{mg} / \mathrm{dl}^{6}$. En niños y en adolescentes finlandenses, usando el método de inmunodifusión radial, se obtuvieron concentraciones de $152 \pm 24,8 \mathrm{mg} / \mathrm{d}$ para apo A-I y $93,6 \pm 21,8$ $\mathrm{mg} / \mathrm{dl}$ para apo $\mathrm{B}^{7}$. En niños rusos, los valores encontrados fueron $147 \pm 22 \mathrm{mg} / \mathrm{dl}$ y $81 \pm 21$ mg/dl de apolipoproteína A-I y $B$ respectivamente, utilizando el método de electroinmunoensayo 8 .

La comparacion de estos resultados es compleja, pues la determinación de apolipoproteinas con métodos inmunológicos es difícil de aplicar, ya que éstos se basan en técnicas dependientes de anticuerpos policlonales (los que pueden no ser totalmente reproducibles de un laboratorio a otro o bien reconocer diferentes epítopes en la misma molécula de apolipoproteína), cuyos resultados -a menudo- son expresados en unidades arbitrarias no comparables. La principal dificultad en la medición inmunológica de apolipoproteínas es la estandarización de los métodos'. Independiente de lo anterior, en conjunto podemos tener una visión aproximada de la concentración de dichas apolipoproteínas en niños y adolescentes.

La mayor parte del colesterol plasmático, 70 a $80 \%$, es transportado mediante la LDL en individuos sanos en ayunas, los 20 a $30 \%$ restantes se distribuyen entre HDL y VLDL. Por su parte, 90\% de las apolipoproteínas A-1 y B se encuentran en la HDL y LDL, respectivamente ${ }^{10}$

La LDL está constituida por colesterol y en $95 \%$ de su parte proteica- por apo $\mathrm{B}$, to que explica la fuerte correlación existente entre ambas. La apolipoproteína B no es transferible,

\section{Tabla}

Distribución en percentiles de las concentraciones séricas (mg/dI) de apolipoproteínas A-I y B en 100 escolares de 5 a 18 años de edad en Concepcion, Chile

\begin{tabular}{lrrrrr}
\hline Percentil & 5 & $\mathbf{1 0}$ & $\mathbf{5 0}$ & $\mathbf{9 0}$ & $\mathbf{9 5}$ \\
\hline Apo A-I & 90 & 95 & 125 & 165 & 170 \\
Apo B & 42 & 45 & 68 & 95 & 105 \\
\hline
\end{tabular}

Apo: apolipoproteínas 
existiendo sólo una molécula de elia por partícula de VLDL, HDL y de LDL. El contenido en colesterol de las HDL, en cambio, es variable, dependiendo -entre otros factores- de su vida media plasmática y de la actividad de las proternas de transferencia entre las lipoproteínas (LCAT y CETP).

La correlación entre el colesterol total y la apolipoproteína es pequeña y concuerda con la falta de correlación entre colesterol total y CHDL en una muestra anterior ${ }^{2}$. A diferencia de la apolipoproteína B, la A-I es transferible y su distribución entre las fracciones de HDL es variable. Algunas partículas contienen apolipoprotcína A-I y A-II, otras sólo A-I. El colesterol HDL representa la suma de ambos tipos sin tener relación con las HDL 2 y HDL 3. Los descensos de apolipoproteína A-I se acompañan invariablemente de descenso de C-HDL, en cambio el contenido de coleșterol de la HDL varía según la transferencia a las lipoproteínas con apolipoproteina B o aumento en la actividad de la lipasa hepática, que reduce el contenido de triglicéridos de las HDL $2^{1}$.

En Bogalusa se encontró una correlación similar (r: 0,39 ) y en niños finlandeses algo menor ( $r: 0,27)$, que en esta muestra ( $r: 0,36$ ) entre colesterol total y apolipoproteína. La correlación entre colesterol total y la apolipoproteína B en los estudios recién mencionados fue r: 0,77 y $r: 0,76$ respectivamente, siendo en la nuestra r: $0,55^{6,7}$.

El coeficiente apo A-I/apo B ha sido utilizado en el rastreo de hiperlipidemia en la infancia. Mayor valor predictivo se otorga al cuociente apo Blapo A-I que superaría el de ambas lipoproteínas y estaría alterado en hijos de padres que han tenido infarto de miocardio ${ }^{12}$. En la detección de dislipidemias los niveles séricos de colesterol total, C-HDL y C-LDL son menos útiles que el cuociente apo B/apo $\mathrm{A}-\mathrm{I}^{13}$. Un ejemplo serían concentraciones elevadas de apo B sin aumento concomitante del C-LDL, en la llamada hiperapobeta lipoproteinemia asociada con el desarrollo de cardiopatía.

El cuociente C-LDL /apo B también es de utilidad en casos de hijos cuyos padres han sufrido infarto al miocardio. En ellos, el estudio de Bogalusa encontró concentraciones menores de apó A-I, y una relación C-LDL/apo $B$ más baja, con relacion apo B/apo $\mathrm{A}$-I mayor que en los sujetos controles. En la muestra de niños de
Concepción, el cuociente C-LDL/apo B resultó algo mayor que en la de Berenson, con niños sin antecedentes, en quienes era $1,11^{4}$.

Aunque los percentiles 5 y 95 se acepten como límites para las concentraciones séricas de las apolipoproteínas en una poblacion aparentemente sana, éstas no necesariamente representan las óptimos con respecto al riesgo de enfermedad coronaria. Ej estudio de Bogalusa reconoce que algunos de sus valores fueron obtenidos en un pequeño número de observaciones y que necesitan datos comparativos de poblaciones pediátricas en diversos lugares geográficos y ámbitos culturales 6 .

Las ventajas de la determinación de las apolipoproteínas para estudiar factores de riesgo cardiovascular son varias: la medición del CHDL requiere la precipitación química y compleja de las fracciones lipoproteicas con su inherente error potencial; la concentración de $\mathrm{C}$ LDL se calcula indirectamente mediante la fórmula de Friedewaid, en cambio, las apolipoproteínas se determinan por un método directo inmunológico, sin tratamiento previo de la muestra y cada una en forma independiente. Por otra parte, las concentraciones de colesterol sérico son fluctuantes, dependiendo -entre otros- del estado nutricional y el ritmo circadiano, lo que no ocurre con las apolipoproteínas?

Aunque se le reconoce valor diagnóstico a la determinación de colesterol sérico total y sus fraccíones, éstas no bastan para identificar en forma inequívoca a los individuos en riesgo cardiovascular. En este punto, las concentraciones de las apolipoproteínas han demostrado poseer un mayor valor predictivo para la evaluación de dicho riesgo.

\section{Resumen}

Se determinó la distribución de las concentraciones séricas de apolipoproteína A-I y apolipoproleína $B$ en una muestra de 100 escolares de 5 a 18 años de edad ( 52 varones y 48 niñas), elegidos aleatoriamente de un universo de 2200 escolares previamente estudiados en la ciudad de Concepción, Chile. El método empleado fue inmunonefelométrico. La distribución de frecuencia para ambas apolipoproteínas fue normal. Los promedios de apolipoproteína A-I fueron $130 \pm 26 \mathrm{mg} / \mathrm{dl}$, los de apolipoproteína B 70 
$\pm 21 \mathrm{mg} / \mathrm{dl}$, mientras que las relaciones fueron apolipoproteína B/ apolipoproteína A-] $=0,70$ : apolipoproteína $A$-Vapolipoproteína $B=1,96 \mathrm{y}$ LDL/apolipoproteína $B=1,56$. En cuanto a la distribución en percentiles, para la apolipoproteína A-I los percentiles 5 y 95 fueron 90 y $170 \mathrm{mg} / \mathrm{dl}$ y para la apolipoproteína B de 42 y $105 \mathrm{mg} / \mathrm{dl}$, respectivamente. Se insiste en la importancia de contar con valores de referencia para las apolipoproteínas en niños chilenos, útiles en detectar precursores de enfermedad coronaria en la vida adulta.

(Palabras clave: apolipoproteína A-I, apolipoproteína B.)

\section{Referencias}

1. Kwiterovitch $P$ : Bjochemical, clinical. epidemio. logical, generic and pathological data in the pediatric age group relevant to the cholestecol hypothesis. Pediatrics 1986: 778: $349-362$.

2. Mitos C. Casanueva V. Campos R, et al.: Factores de riesgo cardiovascular en una población de escolares chilenos. I Parte: Lípidos séricos en 552 niños y adolescentes de 6-15 años. Rev Cbil Pediatr 1990: 61 67-73.

3. Hamsten A: Apolipoproteins, dyslipidemia and premature coronary heart disease. Acta Med Scand 1988: 223: 389-403.
4. Freedmin D. Srintivasan S, Shear C. Franklin F, Webber $L$, Berenson $G$ : The relation of apolipoproteins $A-1$ and $B$ in children to parental myocardial infarction. N Engl J Med 1986; 315; 721-726.

5. Marinetti GV: En: Disorders of Lipid Metabolism. Plenum Press, New York and London 1990: 75-119.

b. Srinivasan $S$, Freedman $D$, Sharma $C$, Webber $L$, Berentson G: Serum apolipoprotein A-1 and B in 2854 children from a bi racial community: Bogalusa heart study. Pediatrics 1986; 78: 189-200.

7. Solakivi S, Jaakola T, Nikkuri T, ef al.: Atherosclerosis precursors in finnish children and adolescents. Serum apolipoproteins A-1 and B. Acta Paediatr Scand J985; Suppl 318: 119-125.

8. Perova N. Aingorn H, Meleskaya $V$, Dorofeeva $T$, Belokoni N: Plasma lipid and apolipoprotein levels in children hereditarily predisposed to coronary heart disease. Acta Pacdiatr Scand 1988; 77: 559-562.

9. Srinivasan $S$. Foster $T$, Berenson $G$ : Comparability of results by simplified methods for measuring serum lipoproteins, in a pediatric population. Clin Chem I980; 26: 1548-53.

10. Tall $R$ : Plasm lipid transfer proteins. J Lipid Res $1986 ; 27: 367-368$.

11. Fruchars J: Lipoprotcin in heterogenety and its effect on apolipoprotein assays. Scand J Clin Lab Invest 1990; 50: $51-57$.

12. Van Stophowt: Apolipoproteínas A-1 y B. Valores observados de A-I y B en una muestra de 5000 individuos. En: Bio Dossier Laboratorio Behring 1988; 242-244.

13. Alegre B, Miralles A, Marinez $C$ : Utilidad de las apolipoproteía y y de las fracciones de colesterol como marcadores de dislipidemia. Clínica e Investigación Ateroesclerosis 1992; 4: 119-122. 